\title{
Culicoides galliardi et Culicoides bassetorum
}

\section{Espèces nouvelles (Diptères Ceratopogonides) trouvées au Lesotho}

\author{
par J. CALloT, M. KREMER et B. MOLET \\ (Collaboration technique: J.-C. Delecolle) \\ Institut de Parasitologie (Directeur: $\mathrm{P}^{\mathrm{r}} \mathrm{J}$. CALlot), Faculté de Médecine de Strasbourg, \\ 3, rue Koeberlé, F 67000 Strasbourg
}

\begin{abstract}
Résumé
Dans une petite récolte de Culicoïdes faite au Lesotho (Sud de l'Afrique), ont été trouvées deux espèces nouvelles: Culicoides galliardi et Culicoides bassetorum. Le premier est proche de $C$. dekeyseri, de $C$. barrosmachadoi et de $C$. hirsutus, le second est tout à fait original par les dessins des ailes.
\end{abstract}

\section{Summary}

In a capture af Culicoïdes made in Lesotho (South of Africa), two new species were found : C. galliardi and C. bassetorum. The first is closely allied to $C$. dekeyseri, $C$. barrosmachadoi and $C$. hirsutus, the second is quite original by the ornementation of its wings.

Une capture de quelques dizaines de Culicoïdes faite à Morija (Lesotho), altitude $1000 \mathrm{~m}$, par l'un des auteurs le 15 septembre 1969 au piège lumineux a permis de trouver deux exemplaires femelles et, d'autre part, deux mâles et une femelle appartenant à deux espèces nouvelles.

Ce «coup de sonde » laisse espérer un terrain d'investigation des plus intéressants en cette partie de la région éthiopienne. 


\section{Culicoides bassetorum n. sp.}

Espèce d'assez grande taille à ailes très fortement et très particulièrement tâchées. Mâle inconnu.

\section{Femelle.}

TÊTE :

Yeux nus, séparés par un intervalle d'un peu moins d'une fossette. Présence d'une suture au-dessus du poil médian.

Antennes: Proscape et scape brun foncé. Articles courts en forme de tonnelets, articles 11 à 14 renflés à leur base et progressivement plus étroits distalement, article 15 presque cylindrique. Les articles 3 à 8 sont brun clair avec une bande sombre distale, l'article 9 s'assombrit progressivement de la base à la partie distale, les articles 10 à 15 sont entièrement sombres.

RÉPARTITION DES SENSILLES, DES SOIES SENSORIELLES TRANSPARENTES ET LONGUEUR DES ARTICLES DES ANTENNES (EN MICRONS)

\begin{tabular}{|c|c|c|c|c|c|c|c|c|c|c|c|c|c|}
\hline Articles $\ldots$. & 3 & 4 & 5 & 6 & 7 & 8 & 9 & 10 & 11 & 12 & 13 & 14 & 15 \\
\hline Sensilles .... & 7 & 0 & 0 & 0 & 0 & 0 & 0 & 1 & 1 & 1 & 1 & 3 à 4 & 0 \\
\hline $\begin{array}{l}\text { Longues soies } \\
\text { transparentes }\end{array}$ & 2 & 2 & 2 & 2 & 2 & 2 & 2 & 2 & + & + & + & + & + \\
\hline $\begin{array}{l}\text { Petites soies } \\
\text { transparentes }\end{array}$ & 1 & 1 & 1 & 1 & 1 & 1 & 1 & 1 & + & + & + & + & + \\
\hline Longueur ... & 48,3 & 27,3 & 27,3 & 27,3 & 29,4 & 29,4 & 29,4 & 31,5 & 63 & 63 & 60,8 & 60,8 & 85 \\
\hline
\end{tabular}

Longueur totale de l'antenne: 582,5 $\mu$.

Indice antennaire: 1,33.

Palpe: articles allongés, cylindriques. Troisième à peine renflé avec une fossette sensorielle unique, ronde et petite, remplie d'organes en massue. Premier article clair, second brun foncé avec une zone claire à son extrémité, troisième avec une bande claire à la base et l'extrémité, 4 et 5 entièrement sombres.

Longueur des articles ( $1^{\text {er }}$ et $2^{\circ}$ mesurés ensemble) en microns : 90,3; 88,2 ; 27,$3 ; 27,3 \mu$.

Longueur totale : $233 \mu$.

Maxilles et mandibules munis de dents.

Armature cibariale munie en son centre de fines épines réunies en touffe. 


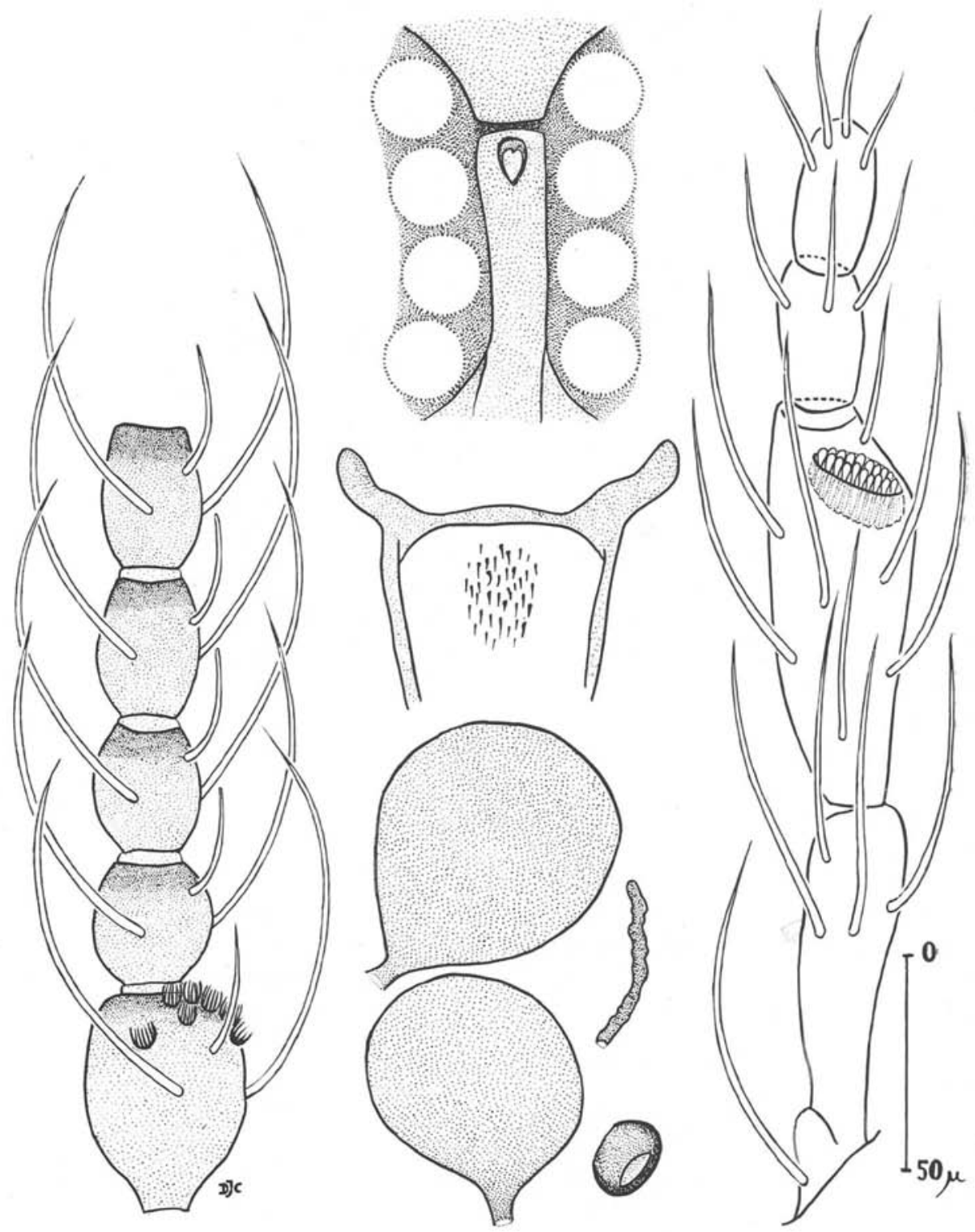

FIG. 1. - Culicoides bassetorum (femelle) : espace interoculaire, palpe, premiers articles antennaires, partie proximale du cibarium, spermathèques 


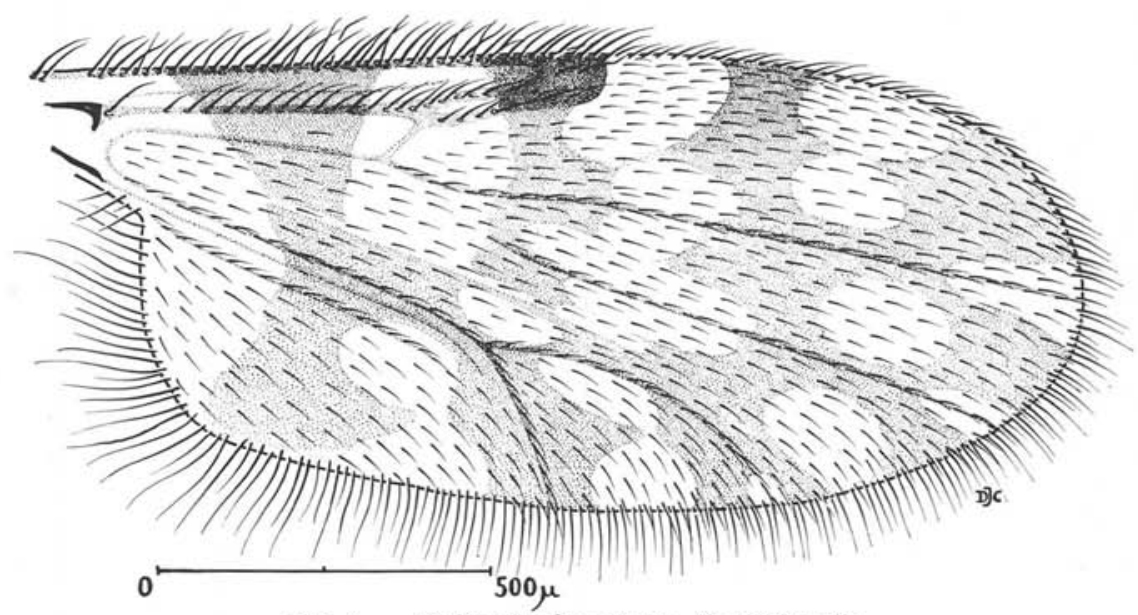

FIG. 2. - Culicoudes bassetorum (femelle) : aile

THORAX :

Thorax non observé (exemplaires conservés en alcool).

Ailes: grises à taches claires bien marquées. Base de l'aile claire, une tache s'étendant du bord de l'aile sur la $\mathrm{T}$, la base de $r 1$ et jusqu'à $m$; une tache dans l'angle $\mathrm{Cu}-\mathrm{Cu} 1$; une succession de taches arrondies, confluentes et chevauchant les nervures s'étendant du bord antérieur, après les radiales jusqu'à $m 2$; une tảche bilobée ou deux taches dans $r 5$, une täche arrondie dans le tiers distal de $m 1$ et $m 2$, une tâche à l'extrémité de M1, M2 et Cu1, une tảche bilobée dans la partie distale de $a n$ et de Cu.

Macrotriches abondantes sur toute la surface de l'aile, mais très rares dans la cellule basale.

Longueur de l'aile : $1,49 \mathrm{~mm}$, largeur : $0,70 \mathrm{~mm}$.

Longueur de la costa: $0,77 \mathrm{~mm}$.

Pattes: fémur antérieur brun avec une zone claire à chaque extrémité, reliées par une bande claire. Médian avec une zone claire à peine visible à chaque extrémité, postérieur avec un léger éclaircissement à sa base. Tibias bruns avec une zone claire à la base et à l'extrémité.

LONGUEUR DES ARTICLES (EN MICRONS)

\begin{tabular}{|c|c|c|c|c|c|c|c|}
\hline & \multirow{2}{*}{ Fémur } & \multirow{2}{*}{ Tibia } & \multicolumn{5}{|c|}{ Tarses } \\
\hline & & & 1 & 2 & 3 & 4 & 5 \\
\hline Pattes antérieures & 401 & 414 & 210 & 112 & 70 & 52,5 & 59,5 \\
\hline Pattes médianes . & 517 & 517 & 276 & 119 & 80,5 & 59,5 & 59,5 \\
\hline Pattes postérieures & 517 & 530 & 255,5 & 136,5 & 91 & 63 & 63 \\
\hline
\end{tabular}

Epines tibiales de: 52,$1 ; 44,1 ; 39,9$ et $35,7 \mu$. 
AbDomen :

Spermathèques arrondies, inégales. Spermathèque rudimentaire très allongée. Anneau sclérifié de $17 \mu$ de large. Longueur des spermathèques 65 et $54,6 \mu$; spermathèque rudimentaire de $40 \mu$ de long.

\section{Discussion}

La disposition toute particulière des taches alaires, notamment, la succession des taches allant du bord antérieur à $m 2$ en chevauchant les nervures est tout à fait typique et cette espèce ne pose pas de problème de diagnose.

\section{Dédicace et dépôt des types}

Cette espèce est très amicalement et très respectueusement dédiée au Docteur Marguerite Basset et au Professeur André Basset.

Type femelle et un exemplaire femelle déposés au laboratoire de Parasitologie de la Faculté de Médecine de Strasbourg.

\section{Culicoides galliardi n. sp.}

Espèce de grande taille, à ailes grises, faiblement tachées de zones claires.

\section{Femelle.}

TÊTE :

Yeux nus séparés par un espace étroit.

Antennes: entièrement brunes. Article 3 claviforme à pédicule court, articles courts renflés à la base, articles longs cylindriques, le dernier atténué distalement.

RÉPARTITION DES SENSILLES, DES SOIES TRANSPARENTES ET LONGUEUR DES ARTICLES (EN MICRONS)

\begin{tabular}{|c|c|c|c|c|c|c|c|c|c|c|c|c|c|}
\hline Articles .... & 3 & 4 & 5 & 6 & 7 & 8 & 9 & 10 & 11 & 12 & 13 & 14 & 15 \\
\hline Sensilles .... & 5 & 2 & 2 & 1 & 1 à 2 & 1 & 1 & 0 à 1 & 1 & 1 & 1 & 3 & 0 \\
\hline $\begin{array}{l}\text { Longues soies } \\
\text { transparentes }\end{array}$ & 2 & 2 & 2 & 2 & 2 & 2 & 2 & 2 & + & + & + & + & + \\
\hline $\begin{array}{l}\text { Petites soies } \\
\text { transparentes }\end{array}$ & 0 & 1 & 1 & 2 & 2 & 2 & 2 & 2 & + & + & + & + & + \\
\hline Longueur .. & 52,5 & 31,5 & 31,5 & 31,5 & 33,6 & 33,6 & 32,5 & 33,6 & 48,3 & 52,5 & 56,7 & 60,8 & 77,7 \\
\hline
\end{tabular}



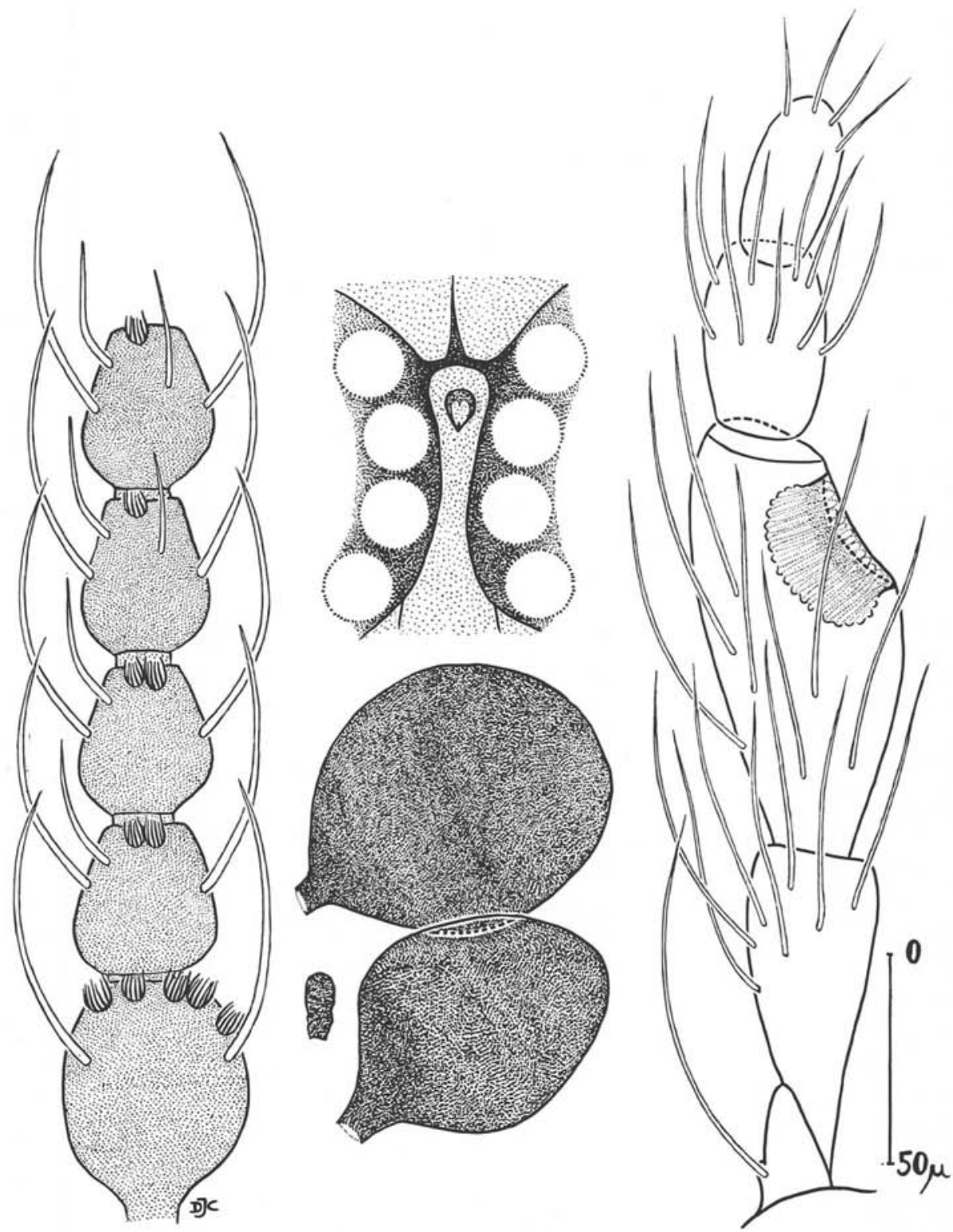

FIG. 3. - Culicoides galliardi (femelle): espace interoculaire, palpe, premiers articles antennaires, spermathèques 
Longueur de l'antenne de $576 \mu$.

Indice antennaire 1,05 à 1,09 (2 antennes).

Palpe: brun, articles cylindriques, trapus, troisième de forme ovale à fossette sensorielle cylindrique profonde.

Longueur des articles: 75,$6 ; 94,5 ; 44,1 ; 35,7 \mu$.

Maxilles et mandibules pourvues de dents.

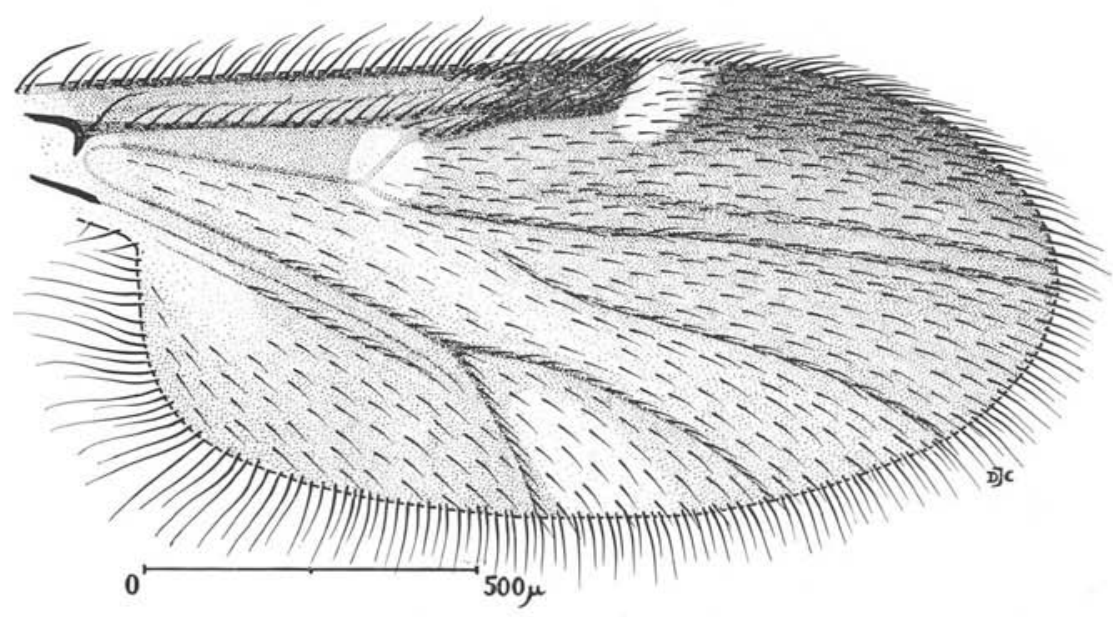

FIG. 4. - Culicoides galliardi (femelle): aile

ThORAX :

Dessins du thorax non observés (exmplaire conservé dans l'alcool).

Ailes: grises, présence d'une tache claire sur la nervure transverse, après la seconde cellule radiale, entre $\mathrm{M}-\mathrm{M} 2$ et $\mathrm{Cu}-\mathrm{Cu} 1$, dans $\mathrm{cu}$, à la base de l'aile et qui se prolonge à la base de an.

Macrotriches abondantes sur toute la surface de l'aile, mais absentes dans la cellule basale et sous-costale.

Longueur de l'aile: $1,45 \mathrm{~mm}$, largeur : 0,67 $\mathrm{mm}$.

Longueur de la costa: $0,82 \mathrm{~mm}$.

Pattes: fémurs bruns à base plus claire. Tibia antérieur à base plus claire, les médians et postérieurs presque entièrement bruns. Genoux noirs. 
LONGUEUR DES ARTICLES DES PATTES DES MÂLES ET DES FEMELLES (EN MICRONS)

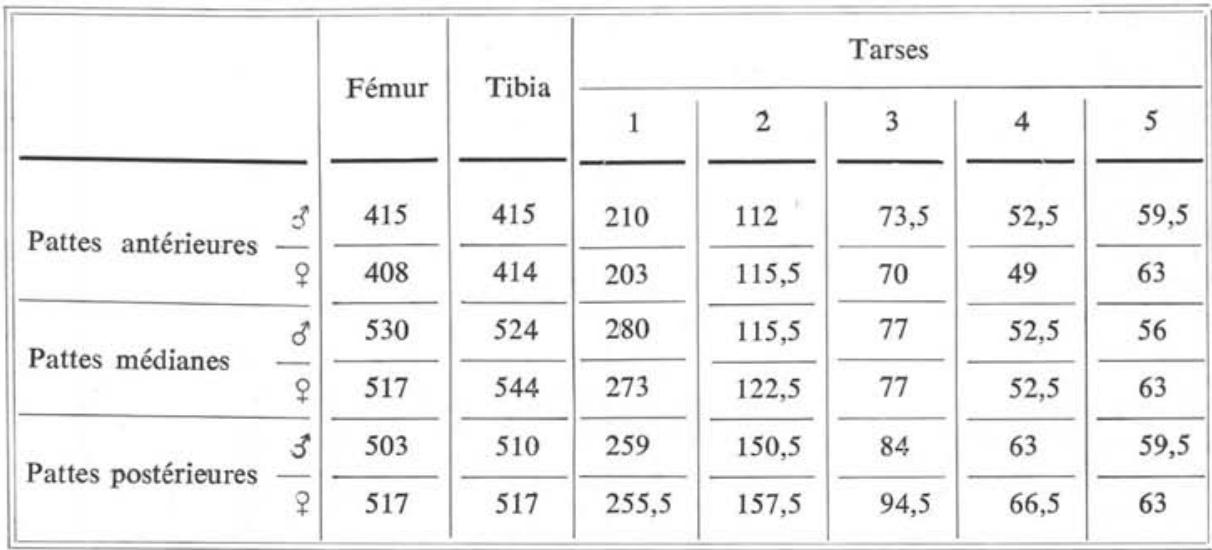

Longueur des épines tibiales de la femelle: 54,$6 ; 63 ; 52,5 ; 46,2 \mu$.

\section{ABdomen :}

Spermathèques sphériques très chitinisées à col de petite dimension.

Longueur des spermathèques : 65 et $75,6 \mu$.

Spermathèque accessoire, épaisse, de $15 \mu$ de long.

Mâle.

TÊTE :

Scape et proscape brun foncé. Articles courts des antennes brun clair, articles longs plus foncés avec une zone plus claire à la base du $13^{\circ}$ et du $14^{\circ}$ articles. Sensilles sur les articles 3, 4, 13 et 14. Deux grandes soies transparentes sur les articles 3 à 6. Une petite soie transparente sur les articles 4 à 12. Palpe brun, articles cylindriques: troisième muni d'une petite fossette sensorielle.

\section{THORAX :}

Ailes comme celles de la femelle, mais taches claires plus importantes. Longueur de l'aile : $1,36 \mathrm{~mm}$, largeur : $0,51 \mathrm{~mm}$.

Longueur de la costa : $0,69 \mathrm{~mm}$.

Pattes: (mensuration: cf. tableau commun aux deux sexes). 


\section{HYPOPYGIUM :}

Lamelle non fendue ou peu fendue. Processus triangulaire. Coxite de forme habituelle à apodème ventral étroit et court et apodème dorsal un peu plus long et large. Sternite coupé par une fente étroite assez profonde. Aedeagus du type de celui de $C$. dekeyseri, présentant une proéminence distale arrondie, corps triangulaire où les bras sont réunis par une membrane fortement chitinisée. Membrane basale fortement spiculée, les spicules remontant presque jusqu'à la partie distale de l'aedeagus. Paramères à base de forme habituelle, prolongée par un corps cylindrique droit comportant des bandes plus chitinisées, qui limitent un espace plus clair ; extrémité aplatie et terminée par deux pointes.

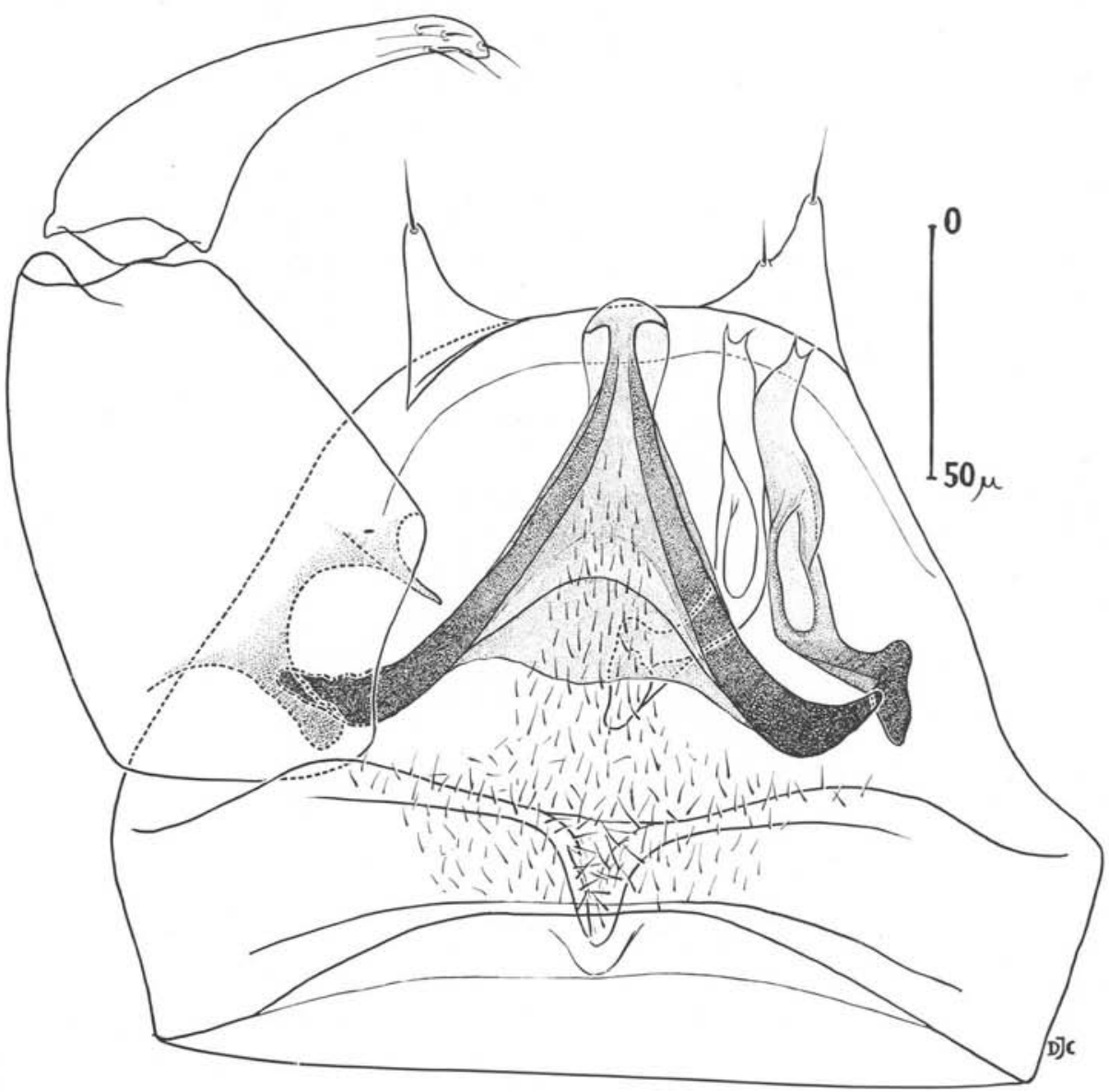

FIG. 5. - Culicoides galliardi (mâle): hypopygium 


\section{Discussion}

C. galliardi se distingue de C. zikaensis et C. barrosmachadoi dont il est proche par sa membrane basale très spiculée et ses ailes peu tâchées.

Il diffère de $C$. dekeyseri par la forme des paramères. Il diffère surtout des deux dernières espèces par la répartition des sensilles et des soies transparentes des antennes.

En effet, $C$. dekeyseri a, chez le mâle, des sensilles sur les articles 3 à 10 et sur le $14^{\circ}$, deux grandes soies transparentes sur les articles 3 à 6 , une sur les articles 7 à 10 , une soie courte sur les articles 4 à 12 .

Le mâle de $C$. barrosmachadoi a des sensilles sur les articles 3,10 et 14 , deux grandes soies transparentes sur les articles 3 à 6 , une grande soie sur les articles 7 à 9 , une soie courte sur les articles 4 à 11 ou 12 .

La femelle est proche de celle de $C$. hirsutus et n'en diffère que par un indice antennaire bien moins élevé $(1,1$ au lieu de 1,44$)$, des spermathèques plus grandes et inégales (65 et 75 au lieu de 45), des épines tibiales dont la seconde est la plus longue.

Cependant, comme le mâle de $C$. hirsutus n'est pas connu, et que les exemplaires sont peu abondants, il est possible qu'il s'agisse de la même espèce et que nous donnions ici la description du mâle de $C$. hirsutus.

\section{Dédicace et dépôt des types}

Nous dédions cette nouvelle espèce à notre Maître, le Professeur Henri Galliard, à l'occasion de son jubilé.

Type mâle et type femelle et un paratype mâle sont déposés au laboratoire de Parasitologie de la Faculté de Médecine de Strasbourg.

\section{Bibliographie}

Callot (J.), Kremer (M.) et Molet (B.), 1967. - Cératopogonidés (Diptères) de la région éthiopienne et particulièrement d'Angola (Description d'espèces et de formes nouvelles). Publ. Cult. Co. Diam. Ang. Lisboa, LxxI, 37-44.

CoRnet (M.). - Communication personnelle.

Clastrier (J.), 1958. - Notes sur les Cératopogonidés. IV, Cératopogonidés d'Afrique occidentale française. Arch. Inst. Pasteur Algérie, XXXVI, 192-258.

Khamala (C. P. M.) et Kettle (D. S.), 1971. - The Culicoides Latreille (Diptera: Ceratopogonidae) of East Africa. Trans. R. ent. Soc. Lond., CXXIII, 1-95. 